\title{
FORMACIÓN UNIVERSITARIA Y PRÁCTICA COMUNITARIA: LA CURRICULARIZACIÓN DE LAS PRÁCTICAS COMUNITARIAS EN UNIVERSIDADES ARGENTINAS
}

\author{
UNIVERSITY TRAINING AND COMMUNITARIAN PRACTICE: \\ COMMUNITARIAN WORK AS PART OF DEGREE CURRICULUM IN \\ ARGENTINIAN UNIVERSITIES
}

\section{FORMAÇÃO UNIVERSITÁRIA E PRÁTICA COMUNITÁRIA: O CURRÍCULO DAS PRÁTICAS COMUNITÁRIAS EM UNIVERSIDADES ARGENTINAS}

\author{
Pedro Gregorio Enriquez* \\ Profesor Titular de la Facultad de Ciencias Humanas en la Universidad Nacional de \\ San Luis \\ Marisol Martín** \\ Profesora en Ciencias de la Educación en la Universidad Nacional de San Luis
}

Resumen: En este trabajo se compara la curricularización de las prácticas comunitarias desarrolladas, en estos últimos años, en 7 (siete) universidades argentinas. Desde lo teórico, las producciones sobre instituciones educativas y sobre reformas curriculares que incluyen el trabajo comunitario, permitieron comprender la complejidad que suponía estos procesos. Desde lo metodológico, el empleo de fuentes documentales digitalizadas (normativas de creación y documentos de apoyo) disponibles en páginas web, posibilitó un primer acercamiento a estas reformas. Como resultado de este estudio se pudo comprobar que los procesos de curricularización generaron cambios en la

\footnotetext{
* Doctor en Pedagogía por la Universidade Aberta do Brasil; Especialista en Metodología de Investigación Científica y Técnica por la Universidad Nacional de Entre Rios.

** Especialista en Constructivismo y Educación por la Facultad Latinoamericana de Ciencias Sociales
} 
universidad, en lo relativo a la forma de relacionarse con la comunidad, en el formato curricular y en la estructura organizacional.

Palabras clave: Práctica comunitaria. Curricularización. Universidad.

Abstract: This article compares communitarian work as part of degree curriculum, developed in the latest years in 7 (seven) Argentinian universities. From a theoretical point of view, productions about educational institutions and curriculum 's reforms that include communitarian work, allows understanding the complexity that these processes suppose. From a methodological perspective, available documentary sources in web pages (creation rules and projects 'supporting documents) make possible to know these reforms. To sum up this study verifies that communitarian work as part of curriculum generates changes in the way universities connect with community, the curriculum format and the organizational structure.

Keywords: Communitarian work. Curriculum. University.

\section{A MODO DE INTRODUCCIÓN}

Las universidades argentinas, a principios del siglo $\mathrm{XX}$, en el marco de la Reforma del '18, inauguraron diversos trabajos comunitarios que conectaban las altas casas de estudios con la sociedad, como parte de las acciones de extensión universitaria. Continuando con esa línea de trabajo, en la década del ' 60 , se generaron distintas experiencias que relacionaban la formación de grado con las demandas de los sectores populares, en ese sentido se destacaron el Programa de Desarrollo Integral de la Isla Maciel (1956-1966) y el Proyecto CEPIA - Centros Pilotos de Investigación Aplicada - (1973-1974) en la Universidad de Buenos Aires (BRUSILOVKY, 2000; LISCHETTI; PETZ, 2009), el Taller Total (1970-1976) en la Facultad de Arquitectura de la Universidad Nacional de Córdoba (ANDER-EGG, 1999), el experimento de pedagogía universitaria participatoria (1973-1974) de la Universidad Nacional de Cuyo (ROIG, 1986), la departamentalización (1974-1976) de la Universidad Nacional de San Luis (MAZZOLA, 2006), entre muchas otras.

No obstante ello, no pudieron masificarse y persistir en el tiempo, porque las dictaduras militares (ONGANÍA 1966-1970; VIDELA et al., 1976-1983) mediante sus políticas autoritarias, conservadoras, elitistas y sus acciones destinadas a expulsar o eliminar físicamente a no-docentes, docentes, alumnos universitarios y diversos actores comunitarios, lograron descartar o diluir estas iniciativas generando un deterioro notable en las condiciones académicas que, aún, resulta difícil de superar. 
Esta situación se está empezando a revertir, en estos últimos años, porque se han tomado algunas decisiones que apuntan a instituir la curricularización de las prácticas comunitarias en diversos espacios formativos del nivel superior.

Así, en 2008, el Consejo Interuniversitario Nacional (CIN) crea la Red Nacional de Extensión Universitaria (Rexuni) como una organización inter-universitaria dependiente de la Comisión de Extensión de este organismo. Entre sus fines se destaca: "Favorecer la institucionalización y valoración de la Extensión Universitaria en todo el ámbito del sistema universitario nacional, promoviendo la inserción curricular y su integración con la docencia y la investigación." (CONSEJO INTERUNIVERSITARIO NACIONAL, 2008).

En 2009, las universidades nacionales convocaron al III Congreso Nacional de Extensión denominado: "La integración, extensión, docencia e investigación. Desafíos para el Desarrollo Social” que se desarrolló en Santa Fe. Uno de los puntos más relevantes fue la incorporación curricular de las prácticas de extensión (proyectos comunitarios, programas territoriales, entre otras) en las carreras universitarias (UNIVERSIDAD NACIONAL DEL LITORAL, 2009).

En 2012 el CIN, mediante el Acuerdo Plenario Nro. 811/12, aprueba el documento elaborado por la Red de Extensión denominado "REXUNI Plan Estratégico 2012-2015" (CONSEJO INTERUNIVERSITARIO NACIONAL, 2012). De las seis líneas definidas como estratégicas, dos involucran a la curricularización de la extensión. La Línea 1, que refiere al reconocimiento académico de la Extensión, recomienda ponderar esta acción en las carreras docentes, del personal de apoyo académico y todas aquellas actividades donde se amerita la trayectoria docente. La Línea 2, que refiere a la promoción de la Inserción Curricular de la Extensión Universitaria, propone promover e incrementar el número de experiencias y desarrollar acciones conjuntas con las áreas académicas que posibiliten esta incorporación.

En este contexto, 7 (siete) universidades argentinas (Universidad Nacional del Litoral - UNL; Universidad Nacional de Río Cuarto - UNRC; Universidad de Buenos Aires - UBA; Universidad Nacional de La Pampa - UNLPam; Universidad de Mar del Plata - UNMdP y las dos Universidades recientemente creadas, la de Avellaneda - UNDAV y la de Rio Negro - UNRN), han elaborado reformas curriculares que incluyen la prácticas comunitarias como un aspecto formativo central.

A los efectos de estudiar comparativamente estas reformas, en este trabajo; primero se hace una presentación general de cada una de ellas y, posteriormente se examina el modo en que las universidades definen su relación con la comunidad, el 
formato curricular que adoptan y la reconfiguración de los aspectos organizacionales que efectúan.

Antes de avanzar en esta dirección es necesario hacer dos observaciones que facilitarán la comprensión de los desarrollos ulteriores.

Primero, este estudio si bien sólo se basa en fuentes documentales digitalizadas (normativas de creación y documentos de apoyo de los proyectos de curricularización) disponibles en páginas web (la mayoría de ellas oficiales), es de suma utilidad en tanto permite comprender las perspectivas y las preocupaciones de quienes elaboraron las reformas; también ayudan a saber, cómo se aprueban, cómo se difunden, a quiénes están destinados, cómo se exige su cumplimiento, etc. No obstante ello es necesario destacar que, los resultados que se obtienen no son concluyentes sino que son indicativos, acotados y provisionales, en tanto refieren únicamente a producciones discursivas que reflejan las miradas de quienes las construyen.

Segundo, este trabajo hunde sus raíces teóricas en los estudios organizacionales y curriculares. Para ello se apela a dos tradiciones investigativas complementarias. Por un lado, se emplea las producciones sobre organizaciones educativas efectuadas por Frigerio, Poggi y Tiramonti (1992) porque permiten examinar los cambios educativos como un campo dinámico que se va re-estructurando permanentemente. Por el otro, se recuperan los trabajos sobre reformas de las curriculas universitarias que incluyen el trabajo comunitario (AROCENA, 2011; TOMMASINO; RODRÍGUEZ, 2011; BOFFELLI; SORDO, 2011; COPELLO, 2010), porque ayudan a comprender cómo, estas prácticas, promueven una relación más abierta con los sectores populares, estimulan el diálogo entre conocimiento científicos con los saberes adquiridos fuera de los ámbitos académicos, configuran nuevas formas organizativas, construyen de nuevos modelos de enseñanza, etc.

\section{DESCRIPCIÓN DE LOS PROYECTOS DE PRÁCTICAS COMUNITARIAS}

En este punto se describen los aspectos generales que encuadran las reformas curriculares que incluyen prácticas comunitarias, desarrolladas por las universidades.

La $U N L$, en el 2007, es la primera universidad argentina que institucionalmente, incorpora las prácticas de extensión en el currículum, en ese marco se desarrolla distintas experiencias formativas con diversos niveles de interacción entre los estudiantes y los sujetos provenientes de diversos sectores sociales. 
Esta universidad, ante la necesidad de impulsar estrategias para vincular la extensión con la formación de grado, crea el Proyecto de Extensión de Cátedra (PEC) que tiene como:

[...] punto de partida el abordaje de una situación problemática a partir de la cual se buscan posibles soluciones para ser implementadas en acciones de transferencia que trasciendan la instancia de análisis y diagnóstico. Estas iniciativas se originan en el seno de cada asignatura, por interés de sus docentes, con el objetivo de integrar las acciones de extensión al proceso pedagógico, posibilitando el aprendizaje de contenidos específicos de los programas académicos, en situación de contexto real. (UNIVERSIDAD NACIONAL DEL LITORAL, 2013).

Como se puede apreciar, su objetivo es vincular la extensión con la actividad académica, permitiendo de ese modo aprender los contenidos en un contexto real, a partir del estudio de una situación-problema y del desarrollo de acciones que tiendan a brindar soluciones a la misma.

La $U N L$ propone dos tipos de prácticas que están incluidas en la formación de grado, las de naturaleza académica integrada a los espacios curriculares que desarrollan prácticas en terreno; y las que se encuentran contempladas en los espacios y/o ciclos de formación profesional (Art. 5. Resol. 274/07). Las prácticas de terreno están ligadas a cualquier espacio curricular que suponga una actuación en el contexto social en general y las profesionales se ligan más a un campo laboral en particular. Esta propuesta no define ni obligatoriedad ni cantidad de horas.

La UNRC en el 2009, mediante Resol. C. S 322/99, aprueba las Prácticas socio-comunitarias (PSC) y las define como: "Actividades de servicio a la comunidad que realizan los estudiantes, supervisados y orientados por los equipos docentes, integradas con los aprendizajes de contenidos (conceptos, procedimientos y actitudes) propios de los espacios curriculares en los que estas prácticas se desarrollan." (UNIVERSIDAD NACIONAL DE RÍO CUARTO, 2010, p. 3). La PSC es obligatoria y se implementa en diversos espacios curriculares. Esta Universidad no fija un crédito horario mínimo.

La $U B A$ en el 2010, mediante Resol. 520/2010, crea la Práctica Social Educativa (PSE) y en 2011 la reglamenta a través de la Resol. 3653/11 donde la define como:

[...] espacios curriculares obligatorios de enseñanza y aprendizaje cuyo desarrollo permite la articulación de contenidos curriculares con necesidades y demandas de la comunidad. Estas propuestas pedagógicas privilegian tanto la adquisición de conocimientos como el beneficio concreto en el campo social atendiendo las necesidades curriculares y de la sociedad, diri- 
gida a sectores en condiciones de vulnerabilidad social, siendo por ello actividades de aprendizaje y de servicio. (UNIVERSIDAD DE BUENOS AIRES, 2011).

Estas prácticas son obligatorias para acceder al título y cuentan con una carga horaria mínima de 42 horas, aunque las facultades pueden incrementar ese horario si lo consideran necesario.

La UNLPam en 2011, mediante la Resol. 297/11, crea el Programa de Práctica Comunitaria (PPC) y lo define como: “[...] espacios de aprendizaje donde se ponen en juego saberes y actitudes para abordar diferentes situaciones de intervención social que propicien el contacto solidario de los estudiantes con la realidad." (UNIVERSIDAD NACIONAL DE LA PAMPA, 2011). Dichas prácticas se implementarán en forma voluntaria en todas las carreras de grado durante los años 2012 - 2016, tornándose obligatorias en el año 2017, momento en que los estudiantes deberán acreditar 40 horas como mínimo para obtener el título de grado.

En la $U N M d P$, las prácticas socio-comunitarias son propuestas que posibilitan una integración entre las actividades de docencia, extensión e investigación, de modo tal que converjan actividades de integración comunitaria con las de aprendizaje de contenidos disciplinares, actividades de investigación de equipos científicos y la elaboración de tesis de posgrado o tesinas de grado (CAUCES EXACTAS, 2013). ${ }^{1}$ Esta universidad exige el cumplimiento de 50 horas reloj como mínimo en todos los planes de estudio.

La UNDAV, como parte del Proyecto Institucional, crea el trayecto curricular integrador denominado Trabajo Social Comunitario (TSC) que tiene como principal objetivo: "[...] producir en el estudiante la construcción de fuertes lazos comunitarios y actitud de involucramiento con la realidad social." (CALZONI, 2011, p. 8). En este trayecto

[...] el desafío central es poder desplegar un trabajo sistemático y sostenido en el tiempo que vaya consolidando la misión de la Extensión universitaria de una vinculación estratégica con la comunidad pero que también aporte desde su proceso de enseñanza-aprendizaje al desarrollo académico y de investigación de estudiantes y docentes. (CALZONI, 2011, p. 2).

El TSC es obligatorio para todos estudiantes y tiene una carga horaria mínima del 7\% (siete) del total de horas de clases presenciales de cada carrera.

En el 2009 momento de su fundación, la UNRN mediante su Estatuto provisorio, crea el Programa de Trabajo Social (PTS). Así, en los Art. 67 y 69 obliga tanto a docentes como a estudiantes a participar de estos espacios, además establece 
que los mismos más el personal no docente tendrían que: “[...] acompañar la resolución de problemas de la comunidad, en particular de los sectores más vulnerables, compartiendo y trasmitiendo conocimientos, habilidades y destrezas vinculados a la vida cotidiana y al ejercicio pleno de sus derechos y del desarrollo de la ciudadanía." (UNIVERSIDAD NACIONAL DO RÍO NEGRO, 2008).

En el año 2012, reglamenta la creación del PTS (Resol 8/12), en ese marco se establece explícitamente cinco criterios: es obligatorio para los estudiantes de las licenciaturas o equivalentes, quedando exceptuados los alumnos de carreras cortas o cursos complementarios; sólo pueden llevarlo a cabo aquellos estudiantes que hayan aprobado el 50\% del plan de estudio, teniendo margen de un año para realizarlo después de haber aprobado el resto de las obligaciones académicas; el crédito horario es de no menos de 64 (sesenta y cuatro) y no más de 200 (doscientas) horas efectivas; este espacio no sustituye las prácticas profesionales incluidas en los planes de estudios; y se lo entiende como actividad extracurricular.

\section{COMPARACIÓN DE LOS ASPECTOS ESTRUCTURANTES QUE CONFORMAN LA CURRICULARIZACIÓN DE LA PRÁCTICA COMUNITARIA}

En este punto se compara las reformas curriculares que incluyen la práctica comunitaria en las universidades; para ello y teniendo en cuenta tres de las cuatros categorías teóricas referidas a las dimensiones construidas por Frigerio, Poggi y Tiramonti (1992), se decide estudiar cómo definen su relación con la comunidad, qué formato curricular adoptan y cómo reconfiguran los aspectos organizacionales.

\subsection{RELACIÓN ENTRE UNIVERSIDAD Y COMUNIDAD}

En sentido amplio, la relación universidad-comunidad, refiere a los vínculos que se establecen entre los miembros de la casa de altos estudios y los diversos sujetos que constituyen su entorno socio-cultural. Los tipos de vínculos que se construyen dependen, al menos, de dos factores que subyacen o se explicitan en los documentos: el modo de concebir al sujeto y la forma de entender la comunicación entre estos dos sectores. 


\subsection{SUJETOS DE LA COMUNIDAD CON QUIENES SE TRABAJA}

En los documentos analizados se pudo observar que cada universidad define los sectores sociales, en ese sentido se identifica los siguientes tipos: los sectores vulnerables solamente, los sectores vulnerables en forma privilegiada involucrando también a otros ámbitos sociales, los sectores populares en particular y la sociedad en su conjunto sin definir a priori ningún criterio de diferenciación.

La $U B A$ es la única universidad que, en la Resol. C. S. 3653/11, explicita claramente que la $P S E$ estará dirigida únicamente a los sectores vulnerables. Puede suponerse entonces, que esta práctica estaría destinada exclusivamente a aquellos sectores que no pueden ejercer plenamente sus derechos, porque su situación de desventaja social, se los imposibilita.

La $U N R C$, en dos documentos refiere a los sujetos con los que trabaja; en uno de ellos señala que la $P S C$ debe ser una experiencia de aprendizaje en contextos de vulnerabilidad económica, social o medio-ambiental (UNIVERSIDAD NACIONAL DE RÍO CUARTO, 2013), en el otro agrega que, no sólo debe limitarse a estos ámbitos sino que tiene que ampliarse a otros sectores que no pueden financiar los servicios que presta la universidad. En este sentido resulta ilustrativo citar textualmente el documento de apoyo de este proyecto, que indica:

Las prácticas no se restringen solamente a contextos de pobreza o vulnerabilidad social sino que pueden involucrar servicios que la Universidad presta en diferentes ámbitos, articulados con los aprendizajes de los estudiantes. Tampoco se trata de servicios a instituciones que pueden autofinanciar estas actividades (instituciones privadas, medianas o grandes empresas, etc.). (UNIVERSIDAD NACIONAL DE RÍO CUARTO, 2010, p. 3).

En suma, de lo señalado puede extraerse que esta universidad privilegiaría el trabajo con los sectores vulnerables pero también incluiría todo sujeto que requiera del servicio, siempre y cuando no esté en condiciones de financiarlo.

Para la $U N M d P$, el $P S C$ implica el desarrollo de acciones que contribuyan a la comprensión y resolución de problemas sociales, ambientales, económicos u otros que comprometan a la sociedad en su conjunto, con especial énfasis en los sectores más vulnerables de la comunidad. Por ello es que se plantean trabajar con instituciones educativas, de salud, colegios profesionales, vecinales, pequeñas empresas, cooperativas, organismos públicos, municipalidades, medios de comunicación locales, en orden a constituirse en verdaderas comunidades de aprendizaje (CAUCES EXACTAS, 2013). 
La $U N R N$, al igual que las otras universidades, deja claro en los considerandos y en la definición que, el PTS está destinado a acompañar a la comunidad en general “[...] con especial énfasis a los sectores vulnerables y desprotegidos." (UNIVERSIDAD NACIONAL DE RÍO NEGRO, 2012). A ello agrega los siguientes objetivos específicos: "[...] ampliar las posibilidades de que los conocimientos sean adecuados para enfrentar los problemas de la sociedad, particularmente de los sectores excluidos [...]" (UNIVERSIDAD NACIONAL DE RÍO NEGRO, 2012) y, “Acercar la Universidad a las comunidades para conocer sus necesidades y colaborar con aquellos sectores que más necesidad tengan de esa cooperación.” (UNIVERSIDAD NACIONAL DE RÍO NEGRO, 2012). Como se puede desprender de estos fragmentos, esta universidad ha decidido que los PTS puedan desplegarse en cualquier sector de la sociedad, pero con especial énfasis en los excluidos, desprotegidos y vulnerables porque consideran que los mismos son los que tienen mayor necesidad de este tipo de práctica.

Comparativamente se puede apreciarse que estas universidades privilegian el trabajo con los sectores en condiciones de desventaja social, pero abren la puerta a otros ámbitos de la sociedad. En el caso de la $U N R C$, la puerta es restringida ya que deja fuera a aquellos que pueden autofinanciar esos servicios; en cambio, en la $U N M d P$ y la $U N R N$ la puerta es más amplia en tanto permite entrar a diversas instituciones asociativas en el primer caso y, sin establecer ningún criterio de exclusión en el segundo.

La $U N D A V$ presenta algunas particularidades en torno a la forma de llamar a los sectores con quienes trabaja. Esta universidad, en el Art. 19 de su Estatuto (UNIVERSIDAD NACIONAL DE LA AVELLANEDA, 2013), determina que el TSC estará dirigido al abordaje de las problemáticas sociales, con particular atención en los sectores populares. En la literatura pedagógica, estos sectores son caracterizados como sujetos que, partiendo de su condición de desigualdad social, pueden constituirse en actores históricos capaces de transformar su realidad (PINTO; ZALAZAR, 1999). Esta forma de definirlos, lejos de colocarlos en un plano de debilidad, como lo hacen aquellos que los entienden como vulnerables o desprotegidos, enfatizan desde su potencia como grupo social.

La UNLPam establece que las Prácticas Comunitarias tienen como intención "[...] otorgar beneficios a los ciudadanos de las zonas de inserción de la UNLPam" y "[...] fortalecer vínculos entre la UNLPam y la comunidad -Asociaciones Civiles y Organizaciones Sociales sin fines de lucro, ONG, Cooperativas, e instituciones públicas." (UNIVERSIDAD NACIONAL DE LA PAMPA, 2011). Además agrega que estos Programas se desarrollarán de forma gratuita en dichos ámbitos (UNIVERSIDAD NACIONAL DE LA PAMPA, 2011). 
Estos artículos generan interpretaciones contradictorias que gravitan en la definición de los sujetos. Determinar que el PPC debe estar destinado a todos los ciudadanos de la región, por un lado y, fortalecer los vínculos y garantizar la gratuidad del PPC a las organizaciones no lucrativas, por el otro, produce algunas confusiones. No deja claro si estas experiencias deben incluir a todos los sujetos sin distinción colocando en pie de igualdad a los diversos sectores (conclusión que se deriva del primer objetivo) o, si solamente están dirigidas a algunas organizaciones (conclusión que se infiere del segundo objetivo y del Ar.4).

\subsection{LA COMUNICACIÓN ENTRE UNIVERSIDAD Y COMUNIDAD}

Siguiendo a Kaplún (1998) existen, al menos, dos modos diferentes de concebir la comunicación. El unidireccional que es de carácter paternalista porque el mensaje fluye en una sola vía, del emisor al receptor y; el bidireccional, por el contrario es dialógica en tanto que emisor y receptor son protagonistas y el mensaje transita por un canal que favorece el proceso de ida y vuelta.

La información proporcionada por los documentos analizados, pone en evidencia los modos predominantes de concebir la comunicación, en ese sentido se puede apreciar que:

La UNLPam, al determinar que en el PPC se debe: “[...] desarrollar una metodología motivadora para la aplicación de los conocimientos adquiridos en el aula." (UNIVERSIDAD NACIONAL DE LA PAMPA, 2011) está aceptando un modelo más unidireccional, porque daría por entendido que la universidad es el lugar donde se construyen los conocimientos legítimos y la comunidad quedaría relegada como un escenario donde se aplica dichos conocimientos.

La UNL, al señalar que la universidad debe involucrarse " $[\ldots]$ con la sociedad y el medio, no sólo transfiriendo tecnologías al tejido productivo de bienes y servicios, sino fundamentalmente escuchando, aprendiendo y reflexionando sobre los contenidos de los mensajes y la naturaleza de los problemas sociales." (UNIVERSIDAD NACIONAL DEL LITORAL, 2007), estaría adoptando una comunicación bidireccional. En otras palabras, esta institución tiene que, no sólo dar respuestas científicas a las necesidades del medio social sino también tiene que aprender diversos aspectos del contexto comunitario.

La UNRC se propone: "Enriquecer la actividad académico-científica de la Universidad con su interacción y comunicación con ámbitos de la realidad social." (Resol. 322/09). Con una tónica equivalente, la UBA se plantea: “Desarrollar la cooperación 
y vínculos entre docentes y estudiantes, y entre ellos y la sociedad." (UNIVERSIDAD DE BUENOS AIRES, 2011). En la misma línea, la $U N D A V$ asume la responsabilidad de aportar a los estudiantes conocimientos teóricos-prácticos que posibiliten la interpretación y la transformación de la comunidad (CALZONI, 2011), y a su vez recuperen de la comunidad "[...] los saberes populares que puedan enriquecer los procesos de enseñanza e investigación." (CALZONI, 2011, p. 2). Como se puede apreciar, estas universidades asumen un modo de comunicación bidireccional que implica un doble proceso, la apropiación de conocimientos académicos para interpretar e intervenir en la comunidad y, a su vez, la incorporación de los saberes comunitarios a la universidad.

Por su parte la $U N M d P$ refiere a la comunicación como una moneda con dos caras. En el objetivo general se determina que la PSC tiene como norte extender a la sociedad, los beneficios de la ciencia, la técnica y la cultura (CAUCES EXACTAS, 2013), en esta cara el saber universitario se presenta como portador de bondades que deben ser transmitidas a la comunidad. En un objetivo específico se propone, en los mismos términos que lo hace la UNRC: enriquecer y direccionar la actividad académico-científica de la Universidad con su interacción y comunicación con ámbitos de la realidad social (CAUCES EXACTAS, 2013). En esta otra cara se admite que la propia universidad se beneficiaría también a partir de la interacción con la comunidad.

La UNRN se plantea: "Aplicar los conocimientos adquiridos en el aula y adquirir otros nuevos, así como desarrollar transferencia práctica, desarrollo de capacidades y fortalecimiento de los sectores de la sociedad que serán beneficiarios del PTS.” (UNIVERSIDAD NACIONAL DE RÍO NEGRO, 2012). Además de ello, también se propone ir " $[\ldots]$ compartiendo y transmitiendo conocimientos, habilidades y destrezas” (UNIVERSIDAD NACIONAL DE RÍO NEGRO, 2012). Hablar en términos de aplicar, transferir, trasmitir, adquirir saberes; desarrollar capacidades, sumado al uso de la expresión sectores sociales beneficiarios, reduce a la comunidad a mero receptor y permite pensar que, en el PTS predominaría una comunicación unidireccional. Solamente la referencia a compartir conocimientos conceptuales y procedimentales, abre un pequeño canal por donde el mensaje podría ir y volver. Esta forma de entender la comunicación se distancia del proyecto fundacional de esta institución, que se proponía como meta, mantener a la Universidad en constante comunicación e interacción con su medio, en donde la marco, la producción de conocimientos, de saberes y respuestas a las diversas necesidades se entendía como tarea conjunta entre la Universidad y las distintas organizaciones sociales con las que se relaciona (UNIVERSIDAD NACIONAL DE RÍO NEGRO, 2009). 


\subsection{EL FORMATO CURRICULAR DE LAS PRÁCTICAS COMUNITARIAS ${ }^{2}$}

Los formatos curriculares, en este trabajo, son entendidos como las prescripciones en torno al modo de organizar los saberes y la tarea pedagógica para llevar a cabo la formación proyectada. En los formatos se concretan las decisiones tomadas sobre: denominación del espacio, intencionalidad formativa, tiempo dedicado, obligatoriedad. Cada uno de estos aspectos, serán examinados a continuación.

La UNL, al llamar Proyecto de Extensión de Cátedra, probablemente está buscando construir una sólida relación entre extensión y enseñanza, por ello promueve la realización de trabajos comunitarios, acorde a las características de cada carrera, con la formación de grado.

La UBA, al adoptar la denominación Práctica Social Educativa, quiere destacar que en este espacio se debe desarrollar actuaciones que vinculen lo social con lo educativo, en ese sentido el abordaje de la problemática social debe producir un efecto formativo, que enriquezca tanto a la comunidad como a los sectores vulnerados de la sociedad.

Tanto la UNRC como la UNMdP, al hablar de Práctica Socio-Comunitaria, quieren destacar que, en ese espacio, se tendría que abordar algún tipo de problemanecesidad mediante intervenciones de carácter social que busquen la implicación de sujetos colectivos.

La UNLPam adopta la expresión Programa de Práctica Comunitaria porque trata de remarcar que las intervenciones deberían involucrar un colectivo humano que comparta un espacio y una cierta conciencia de pertenencia y, además, tendrían que ser sistemáticas. Esto último alude a que los elementos que conforman dichas prácticas deberían estar relacionados entre sí y apuntar a conseguir un objetivo determinado.

La UNDAV, al adoptar la denominación Trabajo Social Comunitario, quiere dejar claro que en ese espacio deberían realizarse acciones colectivas que contribuyan a resolver los problemas sociales y, además faciliten el establecimiento de una sólida relación entre universidad y comunidad.

La UNRN, al denominarlo como Programa de Trabajo Social, quiere destacar que se trataría de un conjunto sistemático de acciones individuales o colectivas que deberían resolver los problemas de la comunidad, apuntando a cumplir, de ese modo, la responsabilidad social de la universidad.

Da la impresión que las formas de llamar a estos espacios curriculares no responden a cuestiones meramente terminológicas, sino que sus nominaciones están 
tratando de imprimirle una impronta particular que de identidad a la práctica que se pretende crear.

En lo relativo a la intencionalidad formativa, en los proyectos se puede advertir el planteamiento de, al menos, dos tipos de objetivos: los de formación académico-profesional que se orientan a la enseñanza de los contenidos disciplinares vinculados al desempeño profesional; y los de formación ético-ciudadana que buscan desarrollar una matriz valorativa que le permita a los estudiantes, sensibilizarse frente a los problemas sociales, asumir responsablemente las acciones que realizan y, asimismo promover su participación en la vida cívica de la comunidad.

La UNL se propone, en el objetivo general, promover la realización de espacios de aprendizaje donde se emprenda el estudio de prácticas, saberes y actitudes para abordar profesionalmente diferentes situaciones sociales. Dichos espacios

[...] tienen por objeto aportar a la formación académica y profesional de los estudiantes en el marco de prácticas de intervención social que los ponga en contacto con la realidad y en situación de asumir una visión crítica, transformadora y solidaria del conocimiento que van construyendo en la Universidad. (UNIVERSIDAD NACIONAL DEL LITORAL, 2007).

En los propósitos específicos se compromete a "[...] formar profesionales con capacidad de creación, reflexión crítica y preocupación por la realidad social y por los efectos sociales de su práctica" y, además “[...] formar profesionales críticos de excelente nivel.” (UNIVERSIDAD NACIONAL DEL LITORAL, 2007).

Como se puede apreciar, la formación académico-profesional y la ético-ciudadana están intrínsecamente relacionadas, en tanto se proponen una formación académica de excelencia que, en este caso tiene que ver con un sujeto capaz de crear, reflexionar críticamente, a la vez, ser sensible y transformar los problemas sociales. Desde el punto de vista de este proyecto, las prácticas que posibilitan el contacto con la realidad son el dispositivo que permite ambas formaciones.

La UNRC se compromete, en su objetivo general, a: "[...] construir y afianzar un currículo que coadyuve a la creación de conciencia social y ciudadana, en el marco de una función crítica de la Universidad." Además, en uno de sus objetivos específicos se plantea: "[...] desarrollar actitudes de solidaridad, sensibilidad y compromiso social en los estudiantes, articuladas con el aprendizaje de contenidos de sus respectivos campos profesionales y científicos, a través de su interacción con problemas de la realidad social." (UNIVERSIDAD NACIONAL DE RÍO CUADRO, 2009). Como se puede observar, la formación académico-profesional y la ético-ciudadana estarían intrínsecamente relacionadas y, en ese horizonte, la práctica socio-comunita- 
ria posibilitaría, no sólo la apropiación de los contenidos académicos sino también la construcción de una ciudadanía responsable y consciente de la necesidad de mejorar las condiciones de existencia de la comunidad.

La $U B A$ se plantea un propósito general y tres objetivos específicos que aluden explícitamente a la formación. El general señala: "Lograr que los estudiantes desarrollen habilidades y valores de ciudadanía, adquieran diversas visiones del mundo y alcancen una posición reflexiva frente a la realidad, a través de la participación en la vida social, de nuevas y variadas formas." (UNIVERSIDAD DE BUENOS AIRES, 2011). Los objetivos específicos indican:

[...] promover el desarrollo de valores de ciudadanía fundados en el compromiso social, promover el desarrollo de habilidades afines con el ejercicio profesional, posibilitar miradas reflexivas y críticas sobre la sociedad desde los enfoques propios de las disciplinas; e incentivar el desarrollo del pensamiento crítico y su utilización para la solución de problemas concretos. (UNIVERSIDAD DE BUENOS AIRES, 2011).

Estas intenciones establecen una fuerte conexión entre la formación académico-profesional y la ético-ciudadana. La práctica comunitaria sería el dispositivo que posibilitaría que el estudiante tenga diversas perspectivas de mundo y asuma una posición reflexiva frente a las mismas, enriquecida por los contenidos disciplinares. En ese marco la apropiación de saberes y el desarrollo de habilidades técnicas propias de la carrera, se integra a la capacidad de comprender la realidad social y de asumir una visión reflexiva, crítica y creativa.

La UNLPam establece que el PPC, en el marco de una formación integral, profesional y humanística, tendría que: “[...] desarrollar los valores de ciudadanía, adquirir diversas visiones del mundo y desarrollar una mirada reflexiva de la realidad en la que se halla inmerso (UNIVERSIDAD NACIONAL DE LA PAMPA, 2011); y por ello podría convertirse en una excelente espacio para "[...] formar ciudadanos participativos y solidarios, y simultáneamente desarrollar una metodología motivadora para la aplicación de los conocimientos adquiridos en el aula." (UNIVERSIDAD NACIONAL DE LA PAMPA, 2011). Al igual que las otras universidades, ésta promueve ambos tipos de formación. Así, desde el punto de vista de lo ético- ciudadano se busca que los estudiantes construyan una visión reflexiva, crítica, participativa y solidaria y, desde lo académico se trata que los alumnos puedan aplicar los conocimientos científicos al contexto comunitario.

La $U N M d P$, en el marco de la misión social de la universidad, se propone consolidar un currículo que posibilite la creación de conciencia social y ciudadana de 
los futuros graduados. En ese horizonte, desde la formación ético-ciudadana se plantea: “[...] la incorporación del estudiante a la realidad laboral y social que le permita, como prestador de un servicio social, sensibilizarse, desarrollar y fortalecer valores morales y éticos", además de "[...] desempeñarse con elevada vocación de servicio, característica necesaria del hombre integrante de una sociedad democrática y participativa." (CAUCES EXACTAS, 2013). Y desde la formación académico-profesional se favorece: "[...] la buena enseñanza articulando los conocimientos de sus respectivos campos profesionales con contextos sociales concretos." (CAUCES EXACTAS, 2013). Al parecer, la práctica socio-comunitaria sería el dispositivo fundamental, tanto para la formación ética y ciudadana como para la formación académico- profesional.

La $U N D A V$, por medio del trayecto de TSC busca generar, en el futuro profesional conductas solidarias y compromiso social, inculcando valores que lo transformen en agente de cambio. Estos espacios curriculares tienen como horizonte brindar aportes teórico-prácticos para que los estudiantes estén en condiciones de interpretar la realidad social, a su vez, puedan emplear distintas estrategias de intervención territorial (identificación, evaluación de problemáticas socio-comunitarias y productivas, elaboración y gestión de proyectos y socialización de los resultados, etc.) que contribuyan a la solución de los posibles problemas (CALZONI, 2011).

Adviértase que esta universidad efectúa una fuerte apuesta a la formación ético-ciudadana en tanto pretende que los alumnos se involucren en la vida comunitaria y participen activamente como agentes de cambio, a tal punto que la formación académica y la apropiación de conocimientos están al servicio del estudio y resolución de los problemas de la realidad social.

La UNRN se propone como objetivo de los PTS, desarrollar las capacidades de los futuros profesionales a través de la experiencia práctica para ampliar la conciencia sobre los problemas propios de las sociedades; completar la formación ética de los estudiantes; ampliar las posibilidades de que los conocimientos sean adecuados para enfrentar los problemas de la sociedad, estimulando la sensibilidad, el compromiso social y la responsabilidad de los egresados y, a la vez, adquieran un mejor conocimiento de la realidad (UNIVERSIDAD NACIONAL DE RÍO NEGRO, 2012).

El PTS sería el dispositivo pedagógico que posibilitaría la formación académico profesional, en tanto que espacio donde se transfieren los saberes aprendidos y se valora su pertinencia; y a su vez, también completaría la formación ético-ciudadana en tanto permitiría sensibilizar, tomar conciencia de los problemas sociales y, asumir el compromiso social y la responsabilidad de enfrentarlos. 
Estos aportes demuestran que todas las universidades consideran a las prácticas comunitarias como dispositivos que contribuyen a la formación tanto académica como ético-ciudadana. Las diferencias estarían dadas en el modo de concebir la relación entre ambas, para algunos son interdependientes en tanto la formación ético-ciudadana y la académica están intrínsecamente vinculadas; y para otros, son complementarias porque ambas operan recíprocamente enriqueciendo las perspectivas de los estudiantes.

En torno al tiempo dedicado a la práctica comunitaria existen diversas posiciones. La $U N L$ no define a priori una carga horaria mínima, dado que el PEC responde a la decisión voluntaria de los equipos docentes según sus intereses. La UNRC tampoco define una carga horaria mínima pero autoriza a las Facultades o Carreras a asignar el crédito horario que consideren conveniente; no obstante ello, deja abierta la posibilidad de establecer una carga horaria común para toda la universidad (UNIVERSIDAD NACIONAL DE RÍO CUARTO, 2013). La UNLPam, la UBA, y la UNMdP fijan un crédito horario mínimo de 40, 42 y 50 horas respectivamente. La UNDAV y la $U N R N$ no determinan una cantidad de horas para la realización de esta experiencia, del modo en que lo hacen las otras universidades y prefieren utilizar otros criterios. La primera fija un porcentaje ( $7 \%$ de las horas totales de clase presenciales de cada una de las carreras) y la segunda establece un rango de no menos de 64 y no más de 200 hs. Vale la pena señalar que en este último incluyen el tiempo de reuniones, traslado, producción de informe, etc.

La fijación o no de un crédito horario a priori es una decisión que entraña algunas complejidades que merecen señalarse. Fijar a priori tiene la ventaja de dejar claro desde el principio la cantidad de tiempo que se va a contar para desarrollar la práctica; no obstante, en ocasiones puede operar como un corsé que restringe las posibilidades del trabajo, en tanto no respeta los procesos que se llevan a cabo en la comunidad. No establecer límites temporales a priori, presenta la ventaja de dejar a consideración del equipo de trabajo el desarrollo de la experiencia, conforme a las necesidades curriculares y sociales que vayan emergiendo, aunque entraña el riesgo de dejar el proceso formativo librado a la voluntad de sus actores en un horizonte de incertidumbre. Por último, es necesario indicar que el crédito horario dedicado a esta formación no puede analizarse en forma independiente, sino que debe examinarse en función del proyecto todo, en particular en relación a los objetivos y a las condiciones de la implementación.

La obligatoriedad de esta práctica es un aspecto que ha generado dos posiciones. La $U N L$ no se pronuncia en torno a este punto, en cambio las otras universi- 
dades exigen la acreditación de estas prácticas, como requisito curricular obligatorio para obtener el título universitario. La obligatoriedad o no de la realización de las prácticas comunitarias genera una fuerte disyuntiva; por un lado, si se impone a los estudiantes el trabajo con la comunidad, se correría el riesgo de tornarse un mero requisito formal, desnaturalizando su sentido; por otro lado, si no se impone, podría ocurrir que una importante porción de estudiantes pasen por la universidad, sin conocer como siente, conoce y vive la comunidad.

Vale la pena destacar aquí que la $U N D A V$, es la única que explicita su intención de evitar que el TSC se transforme en una simple formalidad para conseguir el título. Para ello, instrumenta este espacio formativo desde el comienzo de todas las carreras estableciendo la obligación de desarrollar, en las trayectorias de formación académica, diversas actividades que se realizan dentro y fuera de la universidad. Según esta institución, esta decisión permitiría ir otorgando valor y marco institucional a este tipo de experiencia, evitando asimismo su desnaturalización.

\subsection{LOS ASPECTOS ORGANIZATIVOS}

Como se sabe, la creación de conocimiento (investigación), la transmisión-apropiación de los saberes (enseñanza) y la relación con la comunidad (extensión) son tres funciones básicas de las universidades. Cada una de ellas suele corresponderse con una estructura organizativa específica que tiende a generar una "cultura institucional" propia, no pocas veces aislada o incluso en confrontación con las demás (TAPIA, 2008).

A lo largo de la historia se intentaron romper estas fragmentaciones. Así, el modelo humboiliano revolucionó la universidad del siglo XIX al estructurar la formación en base a la relación investigación-docencia. Arocena (2011) señala que enseñanza, investigación y extensión en prácticas integrales al servicio del desarrollo social, constituiría una propuesta aún más revolucionaria, en tanto que está en el corazón del ideal latinoamericano de universidad.

Para lograr desarrollar la curricularización de la práctica comunitaria, las universidades buscan articular las funciones (TOMMASINO; RODRÍGUEZ, 2011) y simultáneamente crean nuevas estructuras que posibiliten tal propósito. A continuación se describen ambos procesos. 


\subsection{ARTICULACIÓN DE LAS FUNCIONES UNIVERSITARIAS}

Las universidades argentinas, a partir del desarrollo de experiencias de prácticas comunitarias, están tratando de articular las diversas funciones básicas de las mismas. En este sentido se pueden diferenciar dos tipos de proyectos: los que articulan solamente la enseñanza con la extensión y los que articulan enseñanza, investigación y extensión.

a) Los que articulan solamente la enseñanza con la extensión.

Mediante el $P E C$, la $U N L$ intenta articular solamente estas dos funciones, y así aparece expuesto en todos los objetivos específicos:

- Favorecer la articulación de las funciones de extensión y de enseñanza ${ }^{1}$ de tal manera que se refuercen y potencien a los efectos de convertirlas en algo valioso para los estudiantes, para la institución y la comunidad.

- Propiciar el desarrollo de actividades de extensión por parte de los equipos docentes y de los estudiantes, desde propuestas propias de las planificaciones de cátedras y asumiendo la perspectiva que le otorga sentido pedagógico a la extensión universitaria.

- Asumir el pasaje de los estudiantes por la práctica de extensión desde la perspectiva de la formación profesional universitaria, de manera de abordar el propósito de la Universidad de formar profesionales con capacidad de creación, reflexión crítica y preocupación por la realidad social y por los efectos sociales de su práctica.

- Propiciar el abordaje, con los actores universitarios, de temáticas que entran en cuestionamiento al momento de proyectar la articulación de la extensión con la enseñanza, esto es: el tipo de graduado que se quiere formar, la forma que en la Universidad - debería adoptar la enseñanza, el lugar de la práctica en la construcción de conocimiento y en la estructura de los planes de estudio.

- Instalar instancias formativas donde se aborden con los docentes temáticas referidas al diseño de propuestas de extensión de cátedra que posibiliten la construcción de prácticas profesionales desde una perspectiva que articule la formación y el ejercicio profesional y al diseño de situaciones de aprendizaje en la extensión que requiere la identificación de estrategias pedagógicas - tipos de contenidos, de experiencias y metodologías- que permitan formar profesionales críticos de excelente nivel.

- Instalar condiciones legales, estructurales y materiales que contribuyan a la configuración de la práctica de extensión en articulación con la enseñanza. (UNIVERSIDAD NACIONAL DEL LITORAL, 2007).

Como se puede ver en estos fragmentos, la articulación de dos de los tres pilares fundamentales de la universidad, constituye la estrategia que adoptó la $U N L$ 
para vincular el aprendizaje académico con los problemas de la comunidad. En ese marco se propone intencionalmente cambiar:

Al estudiante, aportando una formación profesional que estimule su capacidad de reflexión crítica, de creación, de sensibilidad ante los problemas de la realidad y su compromiso, asumiendo los efectos de su acción. Para ello la universidad, además del $P E C$, ofrece a sus estudiantes la asignatura electiva "Extensión Universitaria" donde se aborda el marco teórico para la puesta en marcha de las prácticas de extensión (MALANO et al., 2011).

$\mathrm{Al}$ docente, generando espacios formativos que lo ayuden a diseñar situaciones de aprendizaje en contacto con la realidad, para ello la universidad organiza cursos de posgrado y de extensión a cargo de especialistas en esta temática (UNIVERSIDAD NACIONAL DEL LITORAL, 2013).

A la universidad como institución educativa, generando condiciones legales y materiales que posibiliten el desarrollo de la reforma curricular. En este sentido crean el área de inclusión curricular, además de vincularla con el proyecto de voluntariado universitario, con los “Centros Universitarios Barriales”, entre otros espacios existentes.

Este breve desarrollo, pone de manifiesto que esta universidad, consciente de la complejidad que supone la curricularización de la práctica comunitaria, hila más fino en su implementación, creando diversas condiciones materiales y simbólicas que posibiliten los cambios necesarios para instrumentar este proceso, sin que muera en mera intención.

El Estatuto (UNIVERSIDAD NACIONAL DE AVALLANEDA, 2013) de la $U N D A V$, también enfatiza solamente la relación entre extensión y enseñanza, en ese sentido destaca: “[...] La Extensión Universitaria estará integrada a la formación curricular de los estudiantes [...] a través del trayecto curricular integrado Trabajo Social Comunitario, el cual será planificado, coordinado y articulado entre la Secretaría de Extensión y la Secretaría Académica." (UNIVERSIDAD NACIONAL DE AVALLANEDA, 2013).

El rector de dicha universidad, a la hora de efectuar una mirada retrospectiva del TSC, señala que es

[...] en la articulación de la enseñanza con la extensión que vamos a ser capaces de construir un saber acorde a las necesidades de la comunidad, trabajando sobre la base de la integración del aporte de los docentes, de los estudiantes y de la comunidad; generando y reafirmando el compromiso con el medio y con la institución. (CALZONI, 2011, p. 10). 
Inmediatamente agrega: "De este modo se irá superando la fragmentación social, logrando que la Universidad se apropie del espacio comunitario así como que la comunidad se apropie del espacio Universitario.” (CALZONI, 2011, p. 10).

El TSC, en esta universidad, sería el dispositivo pedagógico pensado para posibilitar la articulación de la enseñanza con la extensión porque garantizaría la mutua apropiación de los espacios universitarios y comunitarios. No obstante ello, el rector reconoce que este proceso mejoraría el compromiso social de los estudiantes si lograra articular y retroalimentar las tres funciones universitarias (CALZONI, 2011).

b) Los que articulan enseñanza, investigación y extensión.

Cinco universidades explicitan su decisión de articular, no sólo dos sino, las tres funciones, en ese sentido, la UNRC y la UNMdP, en el marco de la PSC, se plantean como uno de sus objetivos contribuir (a través de la enseñanza, la investigación y el servicio) a la resolución de problemas sociales críticos; en el mismo sentido, la $U B A$ determina que la $P S E$ sea el medio adecuado para colaborar en la integración de las funciones de enseñanza, investigación y extensión. Si bien la UNLPam y la $U N R N$ no explicitan en sus objetivos esta articulación, sí proponen incorporar la práctica comunitaria; en el primer caso, bajo las modalidades de módulos dentro de proyectos de voluntariado, de extensión o de investigación y; en el segundo caso, cuando la Vicerrectoría autorice la realización de los PTS en proyectos de extensión o de investigación (UNIVERSIDAD NACIONAL DE RÍO NEGRO, 2013). Estas decisiones favorecen cierto grado de articulación de las tres funciones.

\subsection{CREACIÓN DE NUEVAS ESTRUCTURAS ORGANIZATIVAS}

En la actualidad ya no se puede pensar en una universidad como un conjunto de unidades académicas fragmentadas con funciones añadidas; es necesario lograr la integración de la docencia con la extensión y la investigación, y para ello es imprescindible construir una nueva forma de pensar y organizar esta institución (MACCHIARIOLA, 2010). Con ese fin las universidades han propuesto estructuras interdisciplinares y flexibles que posibilitan dicha articulación. Así:

La $U N L$, coherente con su proyecto que articula extensión y enseñanza, crea el Área de Incorporación Curricular, bajo la dependencia de la Secretaría de Extensión, con el objeto de organizar prácticas que contribuyan a la formación académica de los estudiantes, comprometiéndolos con el contexto comunitario.

Además de ello, plantea un conjunto de pautas y mecanismos que contribuyen a implementar dicha articulación que, a continuación se reproducen: 
a - Las Unidades Académicas en colaboración con las Secretarías de Extensión y Académica de la UNL definirán los espacios curriculares necesarios para el desarrollo de las prácticas de extensión.

b - La Universidad a través de Honorable Consejo Superior dispondrá cuatrimestralmente de una nómina de espacios curriculares posibilitadores de prácticas de extensión de cada una de sus carreras de grado de manera de poner en conocimiento de los estudiantes y ofrecerlos como espacios de desarrollo de dicha actividad.

c - Las Secretarías de Extensión y Académica de la UNL brindarán instancias de asesoramiento y de formación para docentes y estudiantes referidas al diseño de propuestas de aprendizaje en la extensión.

d - La Secretaría de Extensión, en tanto dependencia que se ocupa de estrechar lazos de articulación y trabajo asociativo entre la Universidad y el Medio Social, formalizará Convenios de Colaboración con organismos estatales y/o organizaciones e instituciones públicas o privadas, a los efectos de disponer del listado de necesidades y demandas sociales que orienten las prácticas de extensión.

e - Una vez concluido el proyecto de extensión o bien finalizada la práctica de extensión y aprobada la misma por parte del alumno, la Secretaría de Extensión de la UNL extenderá un certificado a los docentes y estudiantes participantes.

f - Todos los alumnos que realicen las prácticas de Extensión, al tratarse de prácticas académicas regladas, contarán con los alcances de la cobertura de seguro que dispone la UNL. (UNIVERSIDAD NACIONAL DEL LITORAL, 2007).

Por su parte, la $U N R C$, también coherente con su decisión de articular las tres funciones, resuelve; por un lado, incorporar la PSC a la órbita del Centro de Investigación, Formación y Desarrollo (CIFOD) sobre Problemáticas Educativas (Resol. 322/09). Dicho centro (conformado por representantes de las facultades, de estudiantes y de la Secretaría de Planeamiento, Académica, Extensión y Comunicación de la Universidad) tiene la responsabilidad de articular y realizar el seguimiento de los proyectos encuadrados como PSC. Por el otro, encomendar a las comisiones curriculares permanentes de cada Facultad, la responsabilidad de:

a) Diseñar propuestas curriculares, de cátedras o inter-cátedras, para la inserción de prácticas comunitarias (asignaturas en las que se incorporan, carga horaria, requisitos para su cursado, etc.) b) Concertar los convenios y/o protocolos de trabajo que fueran necesarios para iniciar progresivamente la implementación de las experiencias

c) Implementar acciones de formación de los grupos de trabajo -seminarios, talleres, análisis de experiencias, etc. (UNIVERSIDAD NACIONAL DE RÍO CUADRO, 2009). 
También le atribuye a las facultades la potestad de fijar las prioridades institucionales, reglamentar los diferentes aspectos vinculados a su implementación y arbitrar los medios para la coordinación institucional de la programación curricular de las prácticas socio-comunitarias (UNIVERSIDAD NACIONAL DE RÍO CUADRO, 2009).

La $U N M d P$ crea un programa específico dedicado a la $P S C$, dependiente de la Unidad Central de dicha universidad y le asigna las siguientes funciones:

- Formación de los recursos docentes responsables de conducir
las prácticas comunitarias en cada Facultad.
- Concretar una base de datos unificada de organizaciones y
proyectos Intervinientes en las prácticas a los efectos de promo-
ver la actividad interdisciplinaria en el abordaje comunitario.
- Colaborar en la gestión de los convenios necesarios para que
las prácticas puedan ser llevadas adelante.
- Llevar un seguimiento formal de los estudiantes y sus discipli-
nas, que estén llevando adelante prácticas en cada organización
o proyecto.
- Elaborar junto a las Unidades Académicas, a través de la In-
vestigación y el debate interdisciplinario, la línea pedagógica
de la Conceptualización previa a la Intervención.
- Elevar Informes sobre las Organizaciones en las que se reali-
zan las intervenciones. (UNIVERSIDAD DE MAR DEL PLA-
TA, 2011).

Es necesario destacar que, el funcionamiento de dicho programa es propuesto por las Secretarías de Extensión Universitaria y Académica, en consulta con todas las Unidades Académicas.

La $U N D A V$, también coherente con la articulación entre extensión y enseñanza, crea la Comisión de Seguimiento y Evaluación del trabajo comunitario, conformada por autoridades de las Secretarías Académica y la de Extensión Universitaria, sumado a ellos, integrantes del Departamento de Ciencias Sociales y de la Vicerrectoría.

La UNLPam no crea una estructura específica para organizar esta práctica, pero asigna responsabilidades a estructuras ya existentes. En este sentido, encomienda a cada Unidad Académica la incorporación de las $P P C$ en las carreras de grado y la construcción de la reglamentación necesaria para su implementación (UNIVERSIDAD NACIONAL DE LA PAMPA, 2011) y, a la Secretaría de Cultura y Extensión Universitaria, la conformación de una base de datos (UNIVERSIDAD NACIONAL DE LA PAMPA, 2011).

La $U N R N$ tampoco crea una estructura específica, porque ya cuenta con una que en sí misma entraña niveles de articulación entre las distintas funciones universitarias. Así la Secretaría de Docencia, Extensión y Vida Estudiantil tiene la función de elaborar los instructivos para la formulación, ejecución y evaluación de los PTS 
y a la Vicerrectoría de Sede le corresponde aprobarlos (UNIVERSIDAD NACIONAL DE RÍO NEGRO, 2012).

\section{A MODO DE CONCLUSIÓN}

En el presente trabajo se compararon las reformas curriculares que incorporaron en sus planes de estudios experiencias que combinan aprendizaje y trabajo comunitario, llevadas a cabo por 7 (siete) universidades argentinas, en estos últimos años.

En ese recorrido se ha podido comprobar que los procesos de curricularización de las prácticas comunitarias han implicado la toma de decisiones en torno a la forma de relacionarse con la comunidad, el formato curricular y la estructura organizacional. En este sentido se pudo apreciar que:

a) Las diversas denominaciones que cada universidad adopta para llamar a este espacio, no responde a cuestiones terminológicas sino que está indicando la necesidad que tiene cada institución de darle una impronta particular a su práctica que le confiera una marca de identidad. Es necesario recordar aquí que la $U N L$ emplea el nombre de Proyectos de Extensión de Cátedra (PEC), la UNRC y la UNMdP prefieren hablar de Práctica Socio-Comunitaria (PSC), la $U B A$ se inclina por Práctica Social Educativa (PSE), la UNLPam la llama Programa de Práctica Comunitaria (PPC), la UNDAV utiliza Trabajo Social Comunitario (TSC) y UNRN la nombra como Programa de Trabajo Social (PTS).

b) El modo en que cada universidad define los sujetos tiene particular importancia porque marca, no sólo el tipo de sector social con el que se trabaja, sino aquel que se pretende formar a partir de la práctica comunitaria. En este sentido se pudo ver que algunas reformas sólo definen con quiénes trabajar, mientras que otras además plantean los sujetos que quieren formar. Ahora bien, parece definirse el punto de partida y de llegada sin tener en cuenta el proceso de construcción, que no es lineal sino dialéctico, en tanto que los sujetos son seres humanos que están siendo, haciéndose, deshaciéndose y rehaciéndose permanentemente.

c) El modo en que cada universidad se comunica con la comunidad es de vital importancia, porque permite visibilizar como se construyen los vínculos entre estos dos sectores sociales. En este estudio se pudo observar que son pocas las universidades que adoptan el modo pre- 
dominantemente unidireccional. Este modo, sustentado en una visión paternalista, promueve (impone) como legítimo el saber propio de la elite universitaria y ubica a la alta casa de estudios como "dadora exclusiva" de algún tipo de beneficio a la comunidad. Por el contrario, la mayoría de las universidades adoptan el modo bidireccional, éste está edificado en una concepción dialógica que posibilita el encuentro entre diversos sectores sociales y el enriquecimiento mutuo. En ese marco, la comunicación constituye una oportunidad para definir problemas específicos y construir soluciones colectivas.

d) El formato curricular regula el modo de organizar los saberes y la tarea pedagógica, en ese marco se pudo ver que, todas las universidades transforman algunos aspectos del formato curricular tradicional ampliando los horizontes formativos a partir de estrecha relación con la comunidad, la articulación de lo académico y de lo ético-ciudadano, y el cuestionamiento del uso tradicional de los tiempos.

e) Los aspectos organizativos se ven, asimismo, alterados cuando se inicia un proceso de curricularización. En este estudio se pudo observar que todas las universidades, al decidir articular las diversas funciones, tienen que crear nueva estructuras o redefinir las ya existentes. Esta decisión favorece y potencia estas experiencias, lo que sin lugar a dudas beneficia a las casas de altos estudios y a la comunidad. No obstante ello, la complejidad que supone desarrollar prácticas integrales al servicio del desarrollo social, como lo propone Arocena (2011), requeriría una mayor reestructuración que involucre la cultura institucional, las concepciones epistemológicas, pedagógicas, políticas y el lugar de los estudiantes y la comunidad, entre otras. Todo ello conmovería a la institución universitaria.

En suma, como se ha mostrado en este estudio, la curricularización de las prácticas comunitarias se encuentra en un proceso de construcción y, por lo tanto, no existen resultados concluyentes. Para llegar a ellos, sería necesario realizar nuevas investigaciones y evaluaciones que permitan valorar tanto los procesos como los resultados de estas experiencias.

Notas explicativas:

${ }^{1}$ En el caso de la UNMdP, se utiliza la información extraída del blog de la Agrupación Universitaria Causes Exacta que reproduce el Proyecto de prácticas socio-comunitarias presentado en la universidad.

${ }^{2}$ Las negritas de las citas textuales de las resoluciones señaladas no corresponden al texto original. 


\section{REFERENCIAS}

ANDER-EGG, E. Hacia una pedagogía autogestionaria. Magisterio de Río de la Plata. Buenos Aires, 1999.

AROCENA, R. Hacia la reforma universitaria. La extensión en la renovación de la enseñanza: espacios de formación integral. Universidad de la República. Montevideo-Uruguay, 2011.

BOFFELLI, T.; SORDO, S. Incorporación Curricular de la Extensión en las carreras de grado de la Universidad Nacional del Litoral. ¿Cómo pensar la articulación de las prácticas de extensión con las prácticas de voluntariado universitario? En: CONGRESO IBEROAMERICANO DE EXTENSIÓN UNIVERSITARIA, 21., 2011, Santa Fe. Anales... Santa Fe, Argentina, 2011.

BRUSILOVSKY, S. Extensión universitaria y educación popular. Buenos Aires: Eudeba, 2000.

CALZONI, J. Extensión universitaria para la inclusión social. En: CONGRESO IBEROAMERICANO DE EXTENSIÓN UNIVERSITARIA, 21., 2011, Santa Fe. Anais... Santa Fe, Argentina, 2011.

CAUCES EXACTAS. Proyecto prácticas socio comunitarias de la UNMdP. Disponible en: $<$ http://caucesenexactas.blogspot.com.ar/p/proyecto-practicas-socio-comunitarias.html>. Acceso en: 26 ago. 2013.

CONSEJO INTERUNIVERSITARIO NACIONAL. Acuerdo Plenario n. 681/08. 2008. Disponible en: <http:/www.unc.edu.ar/extension-unc/gestion/documentos/ acuerdo-681-08-creacion-de-la-rexuni.pdf>. Acceso en: 26 agosto 2013.

CONSEJO INTERUNIVERSITARIO NACIONAL. Acuerdo Plenario n. 811/12. 2012. Disponible en: <http://www.rexuni.edu.ar/noticias/acuerdo-plenario-811-12>. Acceso en: 26 agosto 2013.

COPELLO, M. I. Educación-Trabajo-Dignidad: Práctica docente extramuros universitarios-Acciones en Educación Básica. En: SEMINÁRIO INTERNACIONAL DE FORMAÇÃO DE PROFESSORES PARA O MERCOSUL/COE SUL, 18., 2010, Florianópolis. Anais... Florianópolis: Universidade Federal de Santa Catarina, 2010.

KAPLÚN, M. Pedagogía de la comunicación. Madrid: Ediciones De la Torre, 1998

LISCHETTI, M.; PETZ, I. Universidad-sociedad: breve historia de un vínculo. Revista Espacios, Universidad de Buenos Aires, n. 41, p. 130-142, 2009. 
MACCHIAROLA, V. Incorporación de prácticas socio-comunitarias al currículo en la universidad Nacional de Río Cuarto. En: CONGRESO NACIONAL DE EXTENSIÓN UNIVERSITARIA, 4., 2010, Mendoza. Anales... Mendoza: Universidad Nacional de Cuyo, 2010.

MALANO, D. et al. Enseñanza de la Extensión en la UNL: de la Teoría a la Práctica. En: CONGRESO IBEROAMERICANO DE EXTENSIÓN UNIVERSITARIA, 11., 2011, Santa Fe. Anales... Santa Fe, 2011.

MAZZOLA, C. La República Universitaria. Elección directa en la Universidad Nacional de San Luis. San Luis: Nueva Editorial Universitaria, 2006.

ROIG, A. Un experimento de Pedagogía Universitaria Participativa: La reforma de los estudiantes de la Universidad Nacional de Cuyo, Mendoza - Argentina, de los años 1973 - 1974. Universidad Nacional de San Luis, San Luis, 1986.

TAPIA, N. Calidad académica y responsabilidad social: el aprendizaje-servicio como puente entre dos culturas universitarias. En: MARTINEZ, M. (Org.). Aprendizaje servicio y responsabilidad social de las universidades. Octaedro-ICE. Barcelona, 2008.

TOMMASINO, H.; RODRÍGUEZ, N. Tres tesis básicas sobre extensión y prácticas integrales en la Universidad de la República. En: AROCENAS, R. et al. Integralidad: tensiones y perspectivas (CSEAM). Cuadernos de Extensión n. 1. Universidad de la República, Montevideo, 2011.

UNIVERSIDAD DE BUENOS AIRES. Resol. n. 520/10. Creación de la Práctica Social Educativa, Buenos Aires, 2010. Disponible en: <http://www.uba.ar/archivos uba/2010-05-26_520.pdf>. Acceso en: 26 agosto 2013.

UNIVERSIDAD DE BUENOS AIRES. Programa de prácticas sociales educativas. Documento para el debate. Secretaría de Extensión Universitaria y Bienestar Estudiantil. Buenos Aires, 2010.

UNIVERSIDAD DE BUENOS AIRES. Resol. n. 3653/11. Reglamentación de Práctica Social Educativa. Buenos Aires, 2011. Disponible en: <http:/www.uba.ar/ archivos_uba/2011-11-23_res\%203653.pdf>. Acceso en: 26 agosto 2013.

UNIVERSIDAD NACIONAL DE LA AVELLANEDA. Resol. n. 12/13. Estatuto de la Universidad Nacional de Avellaneda. Buenos Aires, 2013. Disponible en: $<$ http:// undav.edu.ar/general/recursos/adjuntos/9058.pdf>. Acceso en: 26 agosto 2013. 
UNIVERSIDAD NACIONAL DE LA AVELLANEDA Trabajo social comunitario: cursada obligatoria. Disponible en: $<$ http:/www.undav.edu.ar/index. php? Idcateg=30\&id=1318>. Acceso en: 26 agosto 2013 .

UNIVERSIDAD NACIONAL DE LA PAMPA. Resol. n. 297/11. Creación y aprobación de reglamento del Programa de Práctica Comunitaria. La Pampa, 2011.

UNIVERSIDAD DE MAR DEL PLATA. ORD. Nro. 1747/11. Inclusión curricular de las Prácticas Socio Educativas o denominación equivalente para todas las carreras de grado y pregrado de la Universidad.Mar del Plata. Buenos Aires, 2011.

UNIVERSIDAD NACIONAL DE RÍO CUARTO. Resol. n. 322/09. Incorporación de las prácticas socio-comunitarias al currículo. Córdoba, 2009. Disponible en: $<$ http://www.eco.unrc.edu.ar/wp-content/uploads/2010/10/Res.322-09.pdf $>$. Acceso en: 26 agosto 2013.

UNIVERSIDAD NACIONAL DE RÍO CUARTO. Proyecto: módulos de prácticas socio-comunitarias (PraSoc). Córdoba, 2009. Disponible en: $<$ http://www.eco.unrc. edu.ar/wp-content/ uploads/2010/10/Res.322-09.pdf>. Acceso en: 27 jun. 2013.

UNIVERSIDAD NACIONAL DE RÍO CUARTO. Documento de Apoyo: Proyecto de Incorporación de Prácticas socio-comunitarias al currículo. Córdoba, 2010. Disponible en: < http://www.unrc.edu.ar/unrc/psc/pdfs/Concep $\% 20$ Practicas $\% 20$ sociocomunitarias.pdf $>$. Acceso en: 27 jun. 2013.

UNIVERSIDAD NACIONAL DE RÍO CUARTO ;Y si el conocimiento entra en acción? Práctica Socio-comunitaria. Aprendiendo de la comunidad. Disponible en: $<$ http://www.unrc.edu.ar/unrc/psc/>. Acceso en: 27 jun. 2013.

UNIVERSIDAD NACIONAL DE RÍO NEGRO. Resol. ME. n. 1597/08. Estatuto provisorio de la Universidad Nacional de Rio Negro. Rio Negro, 2008. Disponible en: < http://repositorio.educacion.gov.ar:8080/dspace/bitstream/handle/123456789/78389/13467.pdf?sequence=>. Acceso en: 27 jun. 2013.

UNIVERSIDAD NACIONAL DE RÍO NEGRO. Resol. ME n. 252/09. Proyecto fundacional de la Universidad Nacional de Rio Negro. Rio Negro, 2009. Disponible en: <http://www.unrn.edu.ar/sitio/index.php/extension>. Acceso en: 27 agosto 2013.

UNIVERSIDAD NACIONAL DE RÍO NEGRO. Resol. n. 18/12. Reglamento de Programa de Trabajo Socio-comunitario. Rio Negro, 2012. Disponible en: $<$ http:// unrn.edu.ar/blogs/disinteriores/files/2012/08/Res-CDEyVE-018-12.pdf $>$. Acceso en: 27 agosto 2013 . 
UNIVERSIDAD NACIONAL DEL LITORAL. Resol. n. 274/07. Incorporación de las prácticas de extensión a las carreras de grado, Santa Fe, 2007. Disponible en: $<$ www.unl.edu.ar/articles/ download/604>. Acceso en: 26 agosto 2013.

UNIVERSIDAD NACIONAL DEL LITORAL. Documento Base del III Congreso Nacional de Extensión Universitaria. Santa Fe, 2009. Disponible en: <www.unsl. edu.ar/ webseu/info-detallada.doc $>$. Acceso en: 26 agosto 2013.

UNIVERSIDAD NACIONAL DEL LITORAL. Proyectos de Extensión de Cátedra (PEC). Disponible en: $<\mathrm{http}: / /$ www.unl.unl.edu.ar/eje/359/ Proyectos_de_Catedra.html>. Acceso en: 26 agosto 2013.

VERCELLINO, S. El aprendizaje en el marco de la resolución de los problemas de la comunidad. Revista Digital REDAF, Año 3, n. 5 p. 23-30, 2015. Disponível em: $<$ http://www.revistadigitalredaf.com.ar>. Acesso em: 01 fev. 2015.

Recebido em 01 de outubro de 2014 Aceito em 27 de maio de 2015

Endereço para correspondência: Pedro Gregorio Enriquez. Avenida 25 de Mayo, 384, 5730, Villa Mercedes, San Luis, Argentina; almeida.malu04@gmail.com 